\title{
In Silico Insights of L-Glutamate: Structural Features in Vacuum and in Complex with Its Receptor
}

\author{
Janneth Gonzalez and George E. Barreto \\ Departamento de Nutrición y Bioquímica, Facultad de Ciencias, Pontificia Universidad Javeriana, Bogotá D.C., Colombia \\ Correspondence should be addressed to Janneth Gonzalez; janneth.gonzalez@javeriana.edu.co
}

Received 15 May 2013; Accepted 5 September 2013

Academic Editor: Hari S. Sharma

Copyright (C) 2013 J. Gonzalez and G. E. Barreto. This is an open access article distributed under the Creative Commons Attribution License, which permits unrestricted use, distribution, and reproduction in any medium, provided the original work is properly cited.

\begin{abstract}
Structural properties of the glutamate in vacuum and in complex with its receptor were analyzed. The analysis was focused on global properties, attempting to characterize features such as overall flexibility and common trends in the conformation set. The glutamate, as other ligands in complex with the receptor, adopts a spatial conformation that corresponds to one of the possible molecular equilibrium states in physiological conditions. The glutamate forms an extended structure for all cases, but the energy of the glutamate round out form is lower than the extended glutamate form. The results showed the glutamate as a flexible molecule, which can easily adapt to different interacting environments, and it can be considered as an approximation to address why glutamate interacts with a great number of molecules.
\end{abstract}

\section{Introduction}

The amino acid L-glutamate is considered the main excitatory neurotransmitter in mammals Central Nervous System (CNS) $[1,2]$. Most of the CNS synapses use glutamate as a fast neurotransmitter, and at least $60 \%$ of the synapses in the human brain are glutamatergic [3]. The glutamate has an important physiological role in many aspects of the normal brain function such as cognition, memory, learning, nervous system development, cellular migration, cellular differentiation, and neural death $[4,5]$. After being released from a presynaptic cell, glutamate diffuses across the synaptic cleft and binds to its specific receptors in the cell membrane of a postsynaptic cell. Once across the synaptic cleft, glutamate is recognized by glutamate receptors from a high variety of other molecules. Although the mechanism of molecular recognition has long been considered in a key-keyhole relationship, short-range forces have been considered as the primary cause of such interactions [6].

The glutamate (L-isomer) causes depolarization and excitation of neurons, but it does so by acting on a variety of receptors. The AMPA/kainate receptors respond to the glutamic acid analogues, $\alpha$-amino-3-hydroxy-5methylisoxazole-4-propionic acid (AMPA) and kainic acid.
Other receptors, N-methyl-D-aspartate (NMDA) receptor, also belong to the ion-channel-linked superfamily, and there is a population of metabotropic receptors, as upon activation they simulate a second messenger transduction system [7].

The behavior of different neurotransmitters has been mainly studied using physiological and pharmacological techniques in both vertebrate and invertebrate brains, and recent techniques in molecular biology have clarified the amino acid sequences of their binding sites [8-16]. However, it is important to note that natural and synthesized analogues with small differences in their chemical structures exert completely different influences and could even have opposite ones. This fact gives rise to an important question: which structural and chemical characteristics are necessary for the ligand to recognize its receptor? The accepted ideas about the molecular recognition pattern are that it occurs because a ligand and its receptor are geared toward each other in a relationship resembling that between a key and a keyhole, so their geometrical structures are determinant.

The glutamate, as other ligands in complex with the receptor, adopts a spatial conformation or tridimensional structure that corresponds to one of the possible molecular equilibrium states in physiological conditions. The conformations adopted by glutamate in complex with the receptor are 
TABLE 1: Selected glutamate receptors solved by X-ray crystallography.

\begin{tabular}{|c|c|c|c|c|c|c|c|}
\hline Subunit & PDB ID & Number of monomers & Seq. length (aa) & Res. $\AA$ & Organism & Expression system & Gene \\
\hline \multirow{2}{*}{ GluR2 } & $1 F T J^{13}$ & 3 & 258 & 1.90 & Rattus norvegicus & Escherichia coli & Gria2 \\
\hline & $2 \mathrm{CMO}^{1}$ & 2 & 257 & 2.65 & Rattus norvegicus & Escherichia coli & Gria2 \\
\hline \multirow{3}{*}{ GluR0 } & $11 \mathrm{II} 5^{15}$ & 1 & 221 & 1.60 & Synechocystis sp. & Escherichia coli & sll1147 \\
\hline & $1 \mathrm{US} 4^{16}$ & 1 & 298 & 1.75 & Thermus thermophilus & Escherichia coli & Prot. \\
\hline & 1US5 $^{16}$ & 1 & 298 & 1.50 & Thermus thermophilus & Escherichia coli & Prot. \\
\hline \multirow{3}{*}{ GluR5 } & $1 \mathrm{TXF}^{17}$ & 1 & 248 & 2.10 & Rattus norvegicus & Escherichia coli & Grik1 \\
\hline & $1 \mathrm{YCJ}^{18}$ & 2 & 251 & 1.95 & Rattus norvegicus & Escherichia coli & Grik1 \\
\hline & $2 \mathrm{~F} 36^{19}$ & 4 & 254 & 2.11 & Rattus norvegicus & Escherichia coli & Grik1 \\
\hline \multirow{2}{*}{ GluR6 } & $1 S 7 Y^{20}$ & 2 & 251 & 1.75 & Rattus norvegicus & Escherichia coli & Grik2 \\
\hline & $1 \mathrm{~S} 50^{21}$ & 1 & 258 & 1.65 & Rattus norvegicus & Escherichia coli & Grik2 \\
\hline MGLUR1 & $1 \mathrm{EWK}^{2}$ & 2 & 448 & 2.20 & Rattus norvegicus & Autographa & Mglurl \\
\hline MGLUR1 & $1 \mathrm{ISR}^{24}$ & 1 & 448 & 4.00 & Rattus norvegicus & Spodoptera frugiperda & Mglurl \\
\hline
\end{tabular}

the result of ligand-receptor interactions at an atomic level. It is important to consider that the allowed conformations for a molecule could have dramatic effects on the molecule activity or reactivity. Therefore, it is necessary to consider all the possible conformations for molecular properties studies.

To assess the structural properties of the glutamate, we analyzed a conformational ensemble. The analysis was focused on global properties, attempting to characterize features such as overall flexibility and common trends in the conformation set. It is important to have in mind that the different conformational analysis can be applied to any collection of molecular conformations. These may be generated by sampling techniques with theoretical structures but can also have an experimental origin, such as NMR models or different X-ray structures of the same molecule (or analogous molecules). In the present study, we calculated the structural conformations of glutamate (a) in complex with receptor and (b) in vacuum form, as an approach for understanding the mechanism of the recognition of a ligand by its receptor.

\section{Materials and Methods}

2.1. Experimental Structures. For this study, we used 22 structures of glutamate receptor monomers from different subunits solved by X-ray crystallography and deposited in PDB [17]. The 22 structures were derived from 7 crystals, which 5 of them belong to ionotropic receptors and 2 belong to metabotropic receptors (Table 1).

To estimate the uncertainty grade in the atomic positions from the selected glutamate structures, we measured the following internal variables for the heavy atoms: (a) bonds length; (b) bond angles; and (c) dihedral angles. The statistical analyses were made with program Statistica v.6.0 (v. 5.5). To make a comparison among structures, we performed a multiple structural alignment with the software VMD for molecular analysis [18].

2.2. Theoretical Structures. Under neutral $\mathrm{pH}$ in solvated conditions, the carboxyl groups and amino groups in glutamate

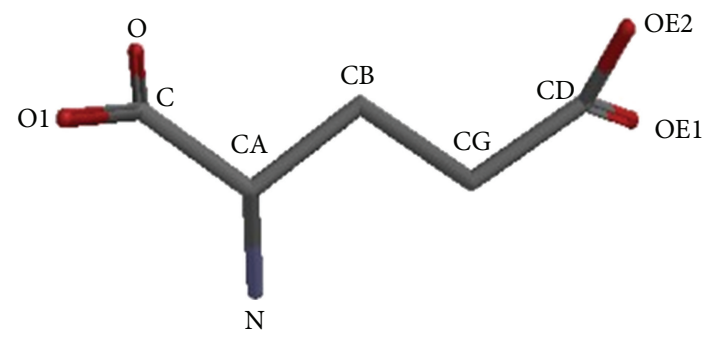

FIgURE 1: Chemical structure of glutamate. The side chain exists in its negatively charged deprotonated carboxylate form at $\mathrm{pHs}$ greater than 4.1; therefore, it is also negatively charged at physiological $\mathrm{pH}$ ranging from 7.35 to 7.45 .

are deprotonated and protonated, respectively. We thus used the ionized models and calculated them in vacuum to obtain the best structural conformation of glutamate. The initial conformation of glutamate was built and optimized with MOE (v. 2007-11) using standard values for the internal 0 variables. The standard nomenclature use by $\mathrm{PDB}$ was considered to point out the heavy atoms in the glutamate molecule (Figure 1).

It is important to note that the number of tridimensional structures expands geometrically with the number of possible combinations for the dihedral angles in the structure. Considering that the conformers differ primarily in their torsion angles, in this study we focused on the conformational space. The exploration of the conformational space was made by changing the dihedral angles in the 30 degrees step (Figure 2).

The database of glutamate conformers used in this study was based on minimal energy glutamate conformations, elimination of duplicated structures, and those with an atomic RMSD value higher than $0.1 \AA$. As a final result, 10 stable structures of glutamate were found. These structures were optimized again by GAMMES program (GAMMES v. Sep 7/2006 (R4)) using RHF/6-31G** approach. Geometry optimization was further performed for the most stable structure of each molecule, and the frequency was then calculated to confirm that the optimized structure was the ground state geometry, using the $6-31 \mathrm{G}^{* *}$ basis set. 


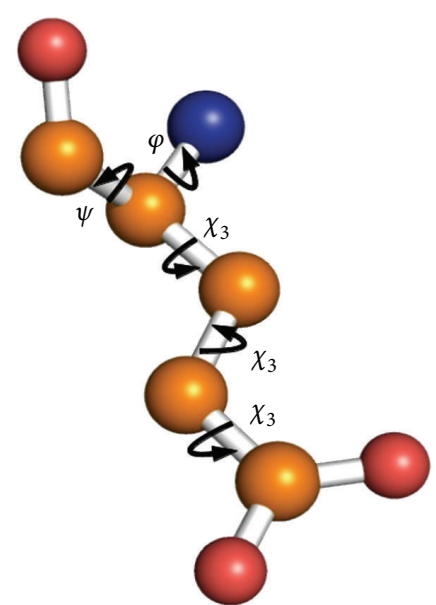

FIGURE 2: Dihedral angles in glutamate. The angles show the main degrees of freedom for the backbone ( $\varphi$ and $\psi$ angles) and the side chain ( $\chi$ angles) of glutamate. The figure shows a ball-and-stick representation of glutamate, which has three $\chi$ angles. The fading conformations in the background illustrate a rotation around $\chi_{1}$.

\section{Results}

3.1. Experimental Structures. It is often assumed that crystal structures have to be obtained at sufficiently high resolution in order to perform macromolecular refinement, and what are considered "acceptable" have been pushed to lower diffraction resolutions. In most cases, the crystal quality is measured according to the resolution value. Although in this study we used two crystals resolved with low resolution, namely, 2CMO (2.65 $\AA$ ) and 1ISR (4.00 $\AA$ ), we found no difference when these structures were compared with the high-resolution structures.

The results show that the average of bond lengths values is not statistically different neither between monomers, which come from the same crystal, nor for monomers coming from different crystals. This result shows that at least for the studied structures, the bond length can be viewed at random, and they do not respond to the fact of coming from different crystals or the resolution degree. According to these results, all structures obtained may be used as models for the following analysis. Table 2 shows the descriptive statistics for bond length variable $(n=22)$.

The variation coefficient is less than $1.00 \%$, and this is consistent with the fact that the maximum range reported for the CG-CD bonds with a value that corresponds to $3.86 \%$ of the all bonds values average [17]. On the other hand, the similarity among bond angles values does not correspond, due to the fact that monomers come from different crystal or experimental resolutions. It can be considered for this variable that all the chosen structures belong to one population with close variations values (Table 3 ).

The variation coefficients higher than $1.00 \%$ are the maximum values in which the $\mathrm{CA}-\mathrm{C}-\mathrm{O}$ angles have an average value of $6,554^{\circ}$. The degree of similarity observed for the internal variables discussed previously (bond lengths and bond angles) was not observed for the dihedral angles. These
TABLE 2: Summary of descriptive statistic for bond length between heavy atoms of glutamate conformers. Variation coefficient is in percentages. The values presented in the table come from monomers from different crystal and different experimental resolutions.

\begin{tabular}{lccccc}
\hline Bond & Average & SD & Min. & Max. & VC (\%) \\
\hline CB-CG & 1,523 & 0,011 & 1,500 & 1,544 & 0,754 \\
CG-CD & 1,525 & 0,013 & 1,509 & 1,568 & 0,873 \\
CD-OE1 & 1,250 & 0,009 & 1,231 & 1,271 & 0,684 \\
CB-CA & 1,535 & 0,010 & 1,524 & 1,576 & 0,671 \\
CA-C & 1,524 & 0,008 & 1,502 & 1,540 & 0,504 \\
C-O & 1,245 & 0,010 & 1,213 & 1,261 & 0,833 \\
CA-N & 1,491 & 0,010 & 1,460 & 1,514 & 0,656 \\
C-O1 & 1,247 & 0,006 & 1,237 & 1,257 & 0,477 \\
CD-OE1 & 1,254 & 0,010 & 1,242 & 1,282 & 0,778 \\
\hline
\end{tabular}

TABLE 3: Summary of descriptive statistic for bond angle between heavy atoms of glutamate conformers. Variation coefficient is in percentages. The values represent monomers from different crystal and different experimental resolutions.

\begin{tabular}{lccccc}
\hline Angle & Average & SD & Min. & Max. & VC (\%) \\
\hline CB-CG-CD & 112,999 & 0,970 & 111,085 & 115,479 & 0,858 \\
CG-CD-OE1 & 118,545 & 1,146 & 114,553 & 120,904 & 0,966 \\
CG-CB-CA & 114,447 & 0,984 & 112,796 & 116,309 & 0,860 \\
CB-CA-C & 110,027 & 0,813 & 108,911 & 112,036 & 0,739 \\
CA-C-O & 118,796 & 1,044 & 117,595 & 121,377 & 0,878 \\
N-CA-C & 111,063 & 1,478 & 108,364 & 114,181 & 1,331 \\
CA-C-O1 & 118,565 & 1,558 & 117,090 & 123,644 & 1,314 \\
CG-CD-OE2 & 119,017 & 1,402 & 117,371 & 123,327 & 1,178 \\
\hline
\end{tabular}

differences are mainly due to the different conformations that glutamate can take in complex with its respective original receptor nearly 22 cases analyzed. This result is a qualitative indicator that the glutamate is a dynamic molecule that shows structural heterogeneity with discrete conformational states that share energetic values in close ranges.

The RMSD value was selected as a quantitative indicator of the structures variability for alternative conformation within the same molecule. The visual analysis of multiple structural alignments allows the identification of three major clusters. These conformations emerged as a result of specific environments of the binding sites interaction with each of the receptors (Figure 3 ).

The first and second groups are composed of 16 and 3 isolated molecules from ionotropic glutamate receptors crystals, and the third group is composed of 3 isolated molecules from metabotropic glutamate receptors crystals. The distribution of clusters based on the RMSD is shown in Table 4 .

After conformational analysis, 22 refined structures were grouped into three clusters based on pairwise main-chain RMSD (Figure 3). Structures in the first cluster (cluster 1) represent $>$ two-thirds of the sample with an RMSD of $0.3 \AA$ RMSD from its center. The second group (clusters 2 and 3) has an RMSD of $2.0 \AA$ from the first cluster center. A structural comparison between minimum energy structures 


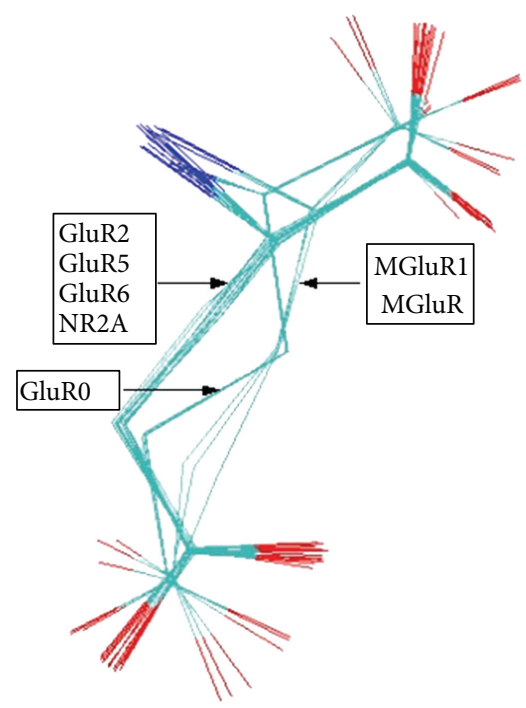

Figure 3: The highest scoring alignment of glutamate. The clusters formed by different conformations are correctly aligned and emerged as a result of specific environments of the binding sites interaction with each of the receptors.

TABLE 4: ID of molecules and receptors from clusters based on pairwise main-chain RMSD. Structures in the first cluster (group 1) represent > two-thirds of the sample with a RMSD of $0.3 \AA$ RMSD. The second cluster (groups 2 and 3) has a RMSD of $2.0 \AA$.

\begin{tabular}{|c|c|c|}
\hline Group & Molecule (ID) & Receptor number \\
\hline \multirow{16}{*}{1} & 1FTJ-274 & \multirow{16}{*}{ GluR2 } \\
\hline & 1FTJ-275 & \\
\hline & 1FTJ-276 & \\
\hline & 2CMO-275 & \\
\hline & 2CMO-274 & \\
\hline & 1TXF-111 & \\
\hline & 1YCJ-998 & \\
\hline & 1YCJ-999 & \\
\hline & 2F36-501 & \\
\hline & $2 \mathrm{~F} 36-502$ & \\
\hline & $2 \mathrm{~F} 36-503$ & \\
\hline & 2F36-504 & \\
\hline & 1S50-999 & \\
\hline & 1S7Y-552 & \\
\hline & 1S7Y-551 & \\
\hline & 2A5S-1001 & \\
\hline \multirow{3}{*}{2} & 1US4-131 & \multirow{3}{*}{ GluR0 } \\
\hline & 1US5-1313 & \\
\hline & 1II5-999 & \\
\hline \multirow{3}{*}{3} & 1EWK-701 & \multirow{2}{*}{ MGluR1 } \\
\hline & 1EWK-702 & \\
\hline & 1ISR-1001 & MGluR \\
\hline
\end{tabular}

of the three clusters is shown in Figure 4. Although it is hard to distinguish clusters by the way that the experimental restraints are satisfied (i.e., by RMSD deviations; see Table 3), we note that the 16 lowest energy structures as evaluated by

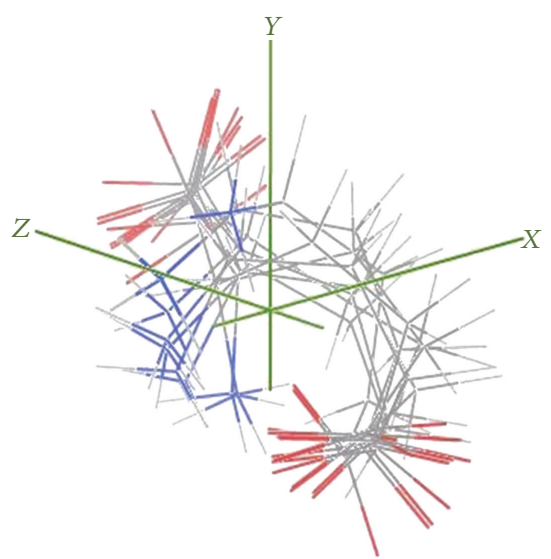

FIGURE 4: Differences between structural alignments. A backbone overlap according to the same alignment is shown; the backbone RMSD is $\leq 0.5$. The figure shows that the conformations adopted by the glutamate are most stable when rounded out.

TABLE 5: The minimum and maximum values for bond angles of the 118 glutamate conformers. The values are in the range from $0.001 \AA$ to $0.02 \AA$.

\begin{tabular}{lccc}
\hline & Min. & Max. & Average \\
\hline C-CA & 1,551 & 1,555 & 1,552 \\
CA-N & 1,498 & 1,504 & 1,501 \\
CA-CB & 1,531 & 1,546 & 1,538 \\
CB-CG & 1,533 & 1,537 & 1,535 \\
CG-CD & 1,528 & 1,533 & 1,530 \\
C-O1 & 1,253 & 1,279 & 1,266 \\
C-O2 & 1,253 & 1,279 & 1,266 \\
CD-OE1 & 1,254 & 1,280 & 1,267 \\
CD-OE2 & 1,254 & 1,280 & 1,267 \\
\hline
\end{tabular}

the MOE scoring function all belong to cluster 1 (structural statistics are given in Table 3).

3.2. Theoretical Structures. The database obtained included 118 structures, which represent locally stable glutamate conformations; neither bond lengths nor bond angles are statistically different, and the values are in a close range $(0.001 \AA$ to $0.02 \AA$ ) for the bond angles of the 118 glutamate conformers. The results are shown in Table 5.

From the comparison of the dihedral angles from the 118 selected structures, we identified 10 groups of conformations with high structural similarity. These 10 groups were found based on different arrangements for multiple structural alignments (RMSD among groups $\geq 1.552$ ).

The conformations included in each group share structural similarity (RMSD $\leq 0.5$ ), and the graphic representation of the structural alignments between selected conformations is shown in Figure 4.

The minimum energy conformations adopted by the glutamate molecule show that the amino acid is most stable when rounded out, despite the extended form that it adopts when interacting with the receptor. This extended conformation 


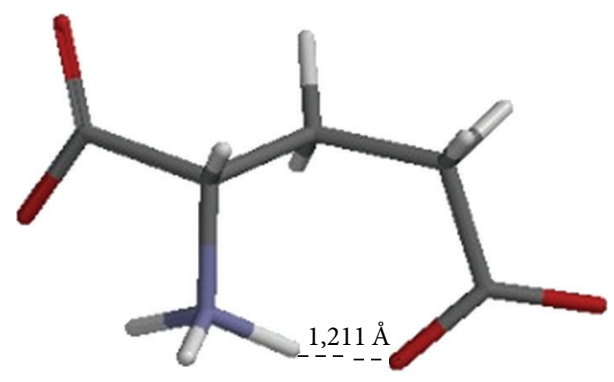

Figure 5: Representation of the glutamate in vacuo shows the propensity to form a hydrogen bond among the amino and carboxyl groups of the principal and side chain, respectively.

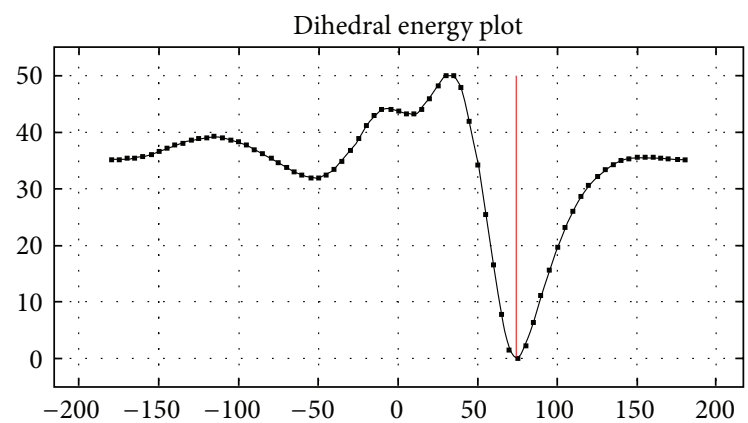

FIGURE 6: Plot of energy versus dihedrals for the selected glutamate conformers. The glutamate structure in free form is determined for the dihedrals angles combinations for which the molecule energy has the lowest values.

is adopted because the lateral chain is bonded to water molecules through hydrogen bonding.

Since the ionized glutamate model in free state is not subjected to interactions with surrounded molecules, it is hypothesized that the rounded form adopted is due to the attraction between the amino and carboxyl side chain groups (Figure 5); the attraction and charge compensation between groups in the same molecule generate a propensity to form a hydrogen bond between these two groups (distance $\mathrm{H}-\mathrm{O}$ $1.211 \AA$ ).

According to the study of the preferential glutamate conformations, it is observed that the maximal variability in the relative position of the heavy atoms is found in the side chain. Figure 6 shows the relationship among the variables energy and dihedrals. The glutamate structure in free form is determined for the dihedrals angles combinations for which the molecule energy has the lowest values.

Although all the conformers obtained share energetic values in a close range (-82.401 y-82,397 Hartrees), the local minimum differs in $1.5 \AA$ (RMSD) average among structures.

\section{Discussion}

The analyses of the classical internal variables of the 22 conformers of glutamate allowed us to identify different groups of conformations according to the type of receptor, which can lead to the idea of the ligand conformation as an indicator of the binding site molecular environment. These results showed the glutamate as a flexible molecule, which can easily adapt to different interacting environments, and it can be considered as an approximation to address why glutamate interacts with a great number of molecules [1-6].

The comparison through the standard method of multiple structures alignment showed that the RMSD values were not dependent on the crystal resolution degree of the diffracted structures. This may be correlated with the standard error of the positional atomic parameters, which can increase if the resolution decreases. Considering that it is not possible to use reliable indicators for the positional standard errors in molecular structures, the use of the RMSD values to compare molecular structures must be considered [19].

The atomic fluctuations of the different conformational states and the structural variability were inherent conditions due to the binding site molecular environment. The molecule flexibility plays a key role in its function [20-23]. Since the RMSD values of the groups obtained are closely related to each other, it was possible to use a representative structural model for each group in order to establish relationships type structural between them for subsequent studies.

The analyses of the RMSD value, according to the glutamate atomic position changes, showed that the variability increased with the atomic distance from the CA. Some hypotheses can be generated from our study: (1) the movement of the principal chain is more conservative in comparison with the side chain if molecule flexibility is considered; (2) the atomic positions of the side chain are variables as the RMSD values show. According to glutamate structure in complex with the receptor binding site reported by Tsuchiya et al. [24] and validated with different subunits of the receptor [8-16], it was found that the glutamate forms an extended structure for all cases, suggesting that there is no number of possibilities to find different geometries when its structure is resolved by X-ray crystallography. The extended form (Figure 3) is different to the round-out form (Figure 4). The energy of the glutamate round out form is lower than the extended glutamate form.

\section{Conclusion}

Glutamic acid is the key amino acid in living organisms. In order to characterize features such as overall flexibility and common trends in the conformation of glutamate in vacuum and in complex with its receptor, we have undertaken theoretical studies. The results showed the glutamate as a flexible molecule, which can easily adapt to different interacting environments, and it can be considered as an approximation to address why glutamate interacts with a great number of molecules; also our results might provide a possible approach for understanding the mechanism of the recognition of glutamate by its receptor.

\section{Conflict of Interests}

The authors declare that there is no conflict of interests. 


\section{Acknowledgment}

This work was supported in part by Grants PUJ 004837, 004247 , and 004960.

\section{References}

[1] J. C. Watkins and R. H. Evans, "Excitatory amino acid transmitters," Annual Review of Pharmacology and Toxicology, vol. 21, pp. 165-204, 1981.

[2] P. M. Headley and S. Grillner, "Excitatory amino acids and synaptic transmission: the evidence for a physiological function," Trends in Pharmacological Sciences, vol. 11, no. 5, pp. 205211, 1990.

[3] R. A. Nichols, T. S. Sihra, A. J. Czernik, A. C. Nairn, and P. Greengard, "Calcium/calmodulin-dependent protein kinase II increases glutamate and noradrenaline release from synaptosomes," Nature, vol. 343, no. 6259, pp. 647-651, 1990.

[4] E. Shimizu, Y.-P. Tang, C. Rampon, and J. Z. Tsien, "NMDA receptor-dependent synaptic reinforcement as a crucial process for memory consolidation," Science, vol. 290, no. 5494, pp. 1170$1174,2000$.

[5] D. E. Berman and Y. Dudai, "Memory extinction, learning anew, and learning the new: dissociations in the molecular machinery of learning in cortex," Science, vol. 291, no. 5512, pp. 2417-2419, 2001.

[6] B. Alberts, A. Johnson, J. Lewis, M. Raff, K. Roberts, and P. Walter, Molecular Biology of the Cell, Garland, New York, NY, USA, 4th edition, 2002.

[7] G. M. Cooper and R. E. Hausman, The Cell: A Molecular Approach, American Society for Microbiology; Washington and Sinauer Associates, Sunderland, Mass, USA, 3rd edition, 2003.

[8] M. Arvola and K. Keinänen, "Characterization of the ligandbinding domains of glutamate receptor (GluR)-B and GluR-D subunits expressed in Escherichia coli as periplasmic proteins," Journal of Biological Chemistry, vol. 271, no. 26, pp. 15527-15532, 1996.

[9] K. Keinänen, A. Jouppila, and A. Kuusinen, "Characterization of the kainate-binding domain of the glutamate receptor GluR6 subunit," Biochemical Journal, vol. 330, no. 3, pp. 1461-1467, 1998.

[10] H. Furukawa and E. Gouaux, "Mechanisms of activation, inhibition and specificity: crystal structures of the NMDA receptor NR1 ligand-binding core," EMBO Journal, vol. 22, no. 12, pp. 2873-2885, 2003.

[11] Y. Paas, M. Eisenstein, F. Medevielle, V. I. Teichberg, and A. Devillers-Thiéry, "Identification of the amino acid subsets accounting for the ligand binding specificity of a glutamate receptor," Neuron, vol. 17, no. 5, pp. 979-990, 1996.

[12] H. Takahashi, E. Inagaki, C. Kuroishi, and T. H. Tahirov, "Structure of the Thermus thermophilus putative periplasmic glutamate/glutamine-binding protein," Acta Crystallographica Section D, vol. 60, no. 10, pp. 1846-1854, 2004.

[13] M. L. Mayer, "Crystal structures of the GluR5 and GluR6 ligand binding cores: molecular mechanisms underlying kainate receptor selectivity," Neuron, vol. 45, no. 4, pp. 539-552, 2005.

[14] P. Naur, B. Vestergaard, L. K. Skov, J. Egebjerg, M. Gajhede, and J. S. Kastrup, "Crystal structure of the kainate receptor GluR5 ligand-binding core in complex with (S)-glutamate," FEBS Letters, vol. 579, no. 5, pp. 1154-1160, 2005.
[15] M. L. Mayer, A. Ghosal, N. P. Dolman, and D. E. Jane, "Crystal structures of the kainate receptor GluR5 ligand binding core dimer with novel GluR5-selective antagonists," Journal of Neuroscience, vol. 26, no. 11, pp. 2852-2861, 2006.

[16] H. Furukawa, S. K. Singh, R. Mancusso, and E. Gouaux, "Subunit arrangement and function in NMDA receptors," Nature, vol. 438, no. 7065, pp. 185-192, 2005.

[17] Protein Data bank, http://www.rcsb.org/pdb.

[18] M. H. Nanao, T. Green, Y. Stern-Bach, S. F. Heinemann, and S. Choe, "Structure of the kainate receptor subunit GluR6 agonistbinding domain complexed with domoic acid," Proceedings of the National Academy of Sciences of the United States of America, vol. 102, no. 5, pp. 1708-1713, 2005.

[19] G. Dodson and C. S. Verma, "Protein flexibility: its role in structure and mechanism revealed by molecular simulations," Cellular and Molecular Life Sciences, vol. 63, no. 2, pp. 207-219, 2006.

[20] J. Chen, N. Sawyer, and L. Regan, "Protein-protein interactions: general trends in the relationship between binding affinity and interfacial buried surface area," Protein Science, vol. 22, pp. 510511, 2013.

[21] A. Q. Zhou, S. C. O’Hern, and L. Regan, “The power of hardsphere models: explaining side-chain dihedral angle distributions of Thr and Val," Biophysical Journal, vol. 102, pp. 23452352, 2012.

[22] D. A. Liberles, S. A. Teichmann, I. Bahar et al., "The interface of protein structure, protein biophysics, and molecular evolution," Protein Science, vol. 21, pp. 769-785, 2012.

[23] A. Q. Zhou, S. C. O’Hern, and L. Regan, “Revisiting the Ramachandran plot from a new angle," Protein Science, vol. 20, pp. 1166-1171, 2011.

[24] D. Tsuchiya, N. Kunishima, N. Kamiya, H. Jingami, and K. Morikawa, "Structural views of the ligand-binding cores of a metabotropic glutamate receptor complexed with an antagonist and both glutamate and Gd3+," Proceedings of the National Academy of Sciences of the United States of America, vol. 99, no. 5, pp. 2660-2665, 2002. 

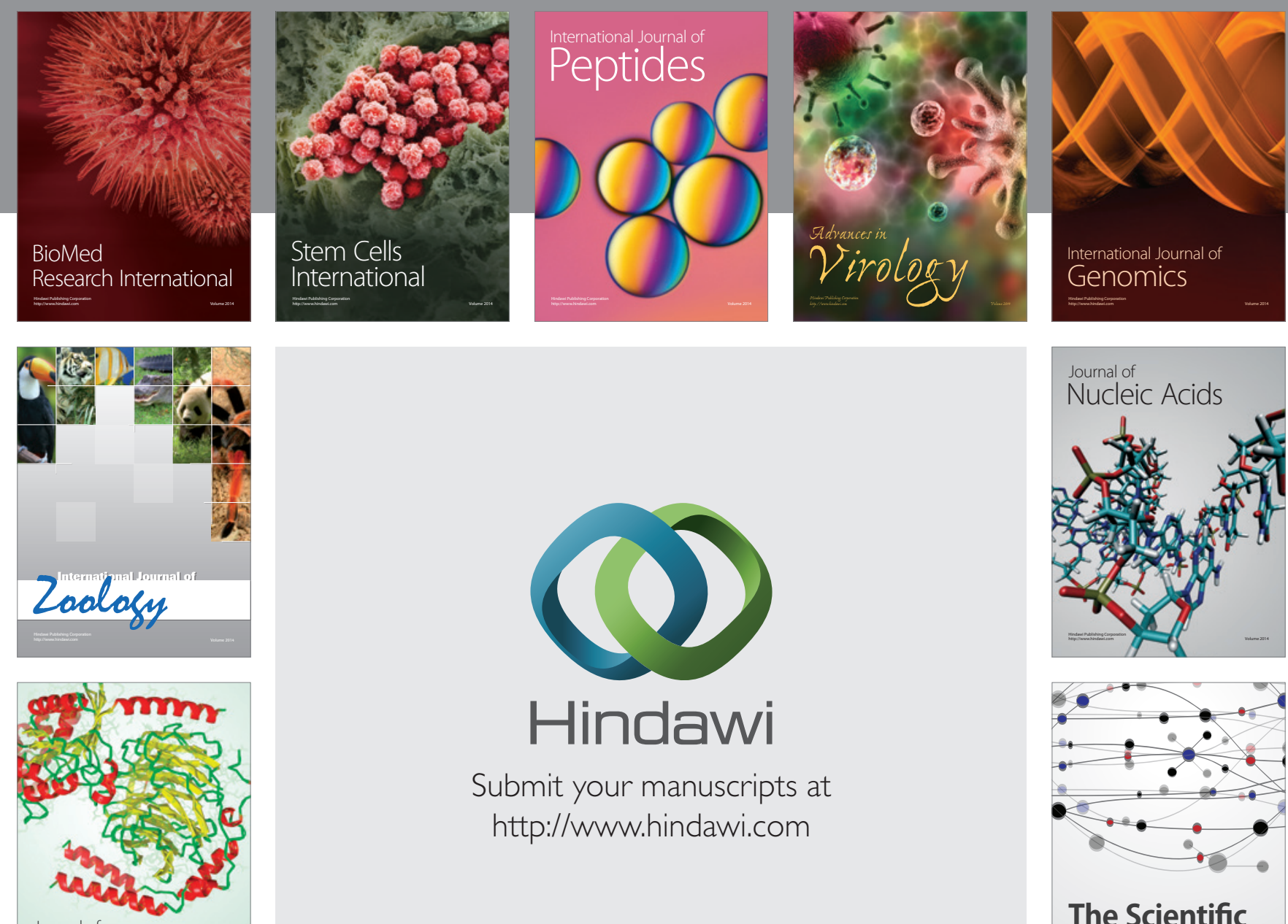

Submit your manuscripts at

http://www.hindawi.com

Journal of
Signal Transduction
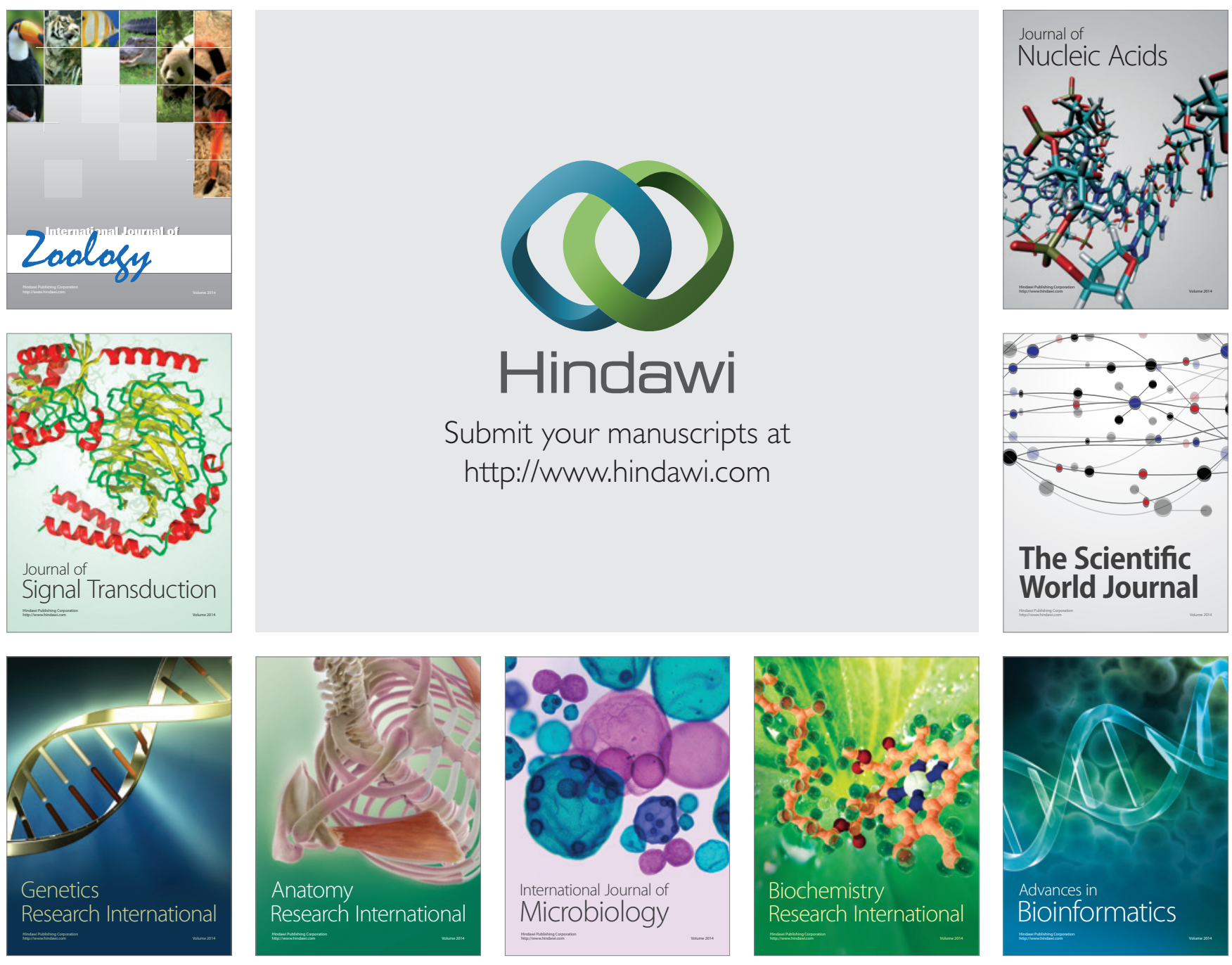

The Scientific World Journal
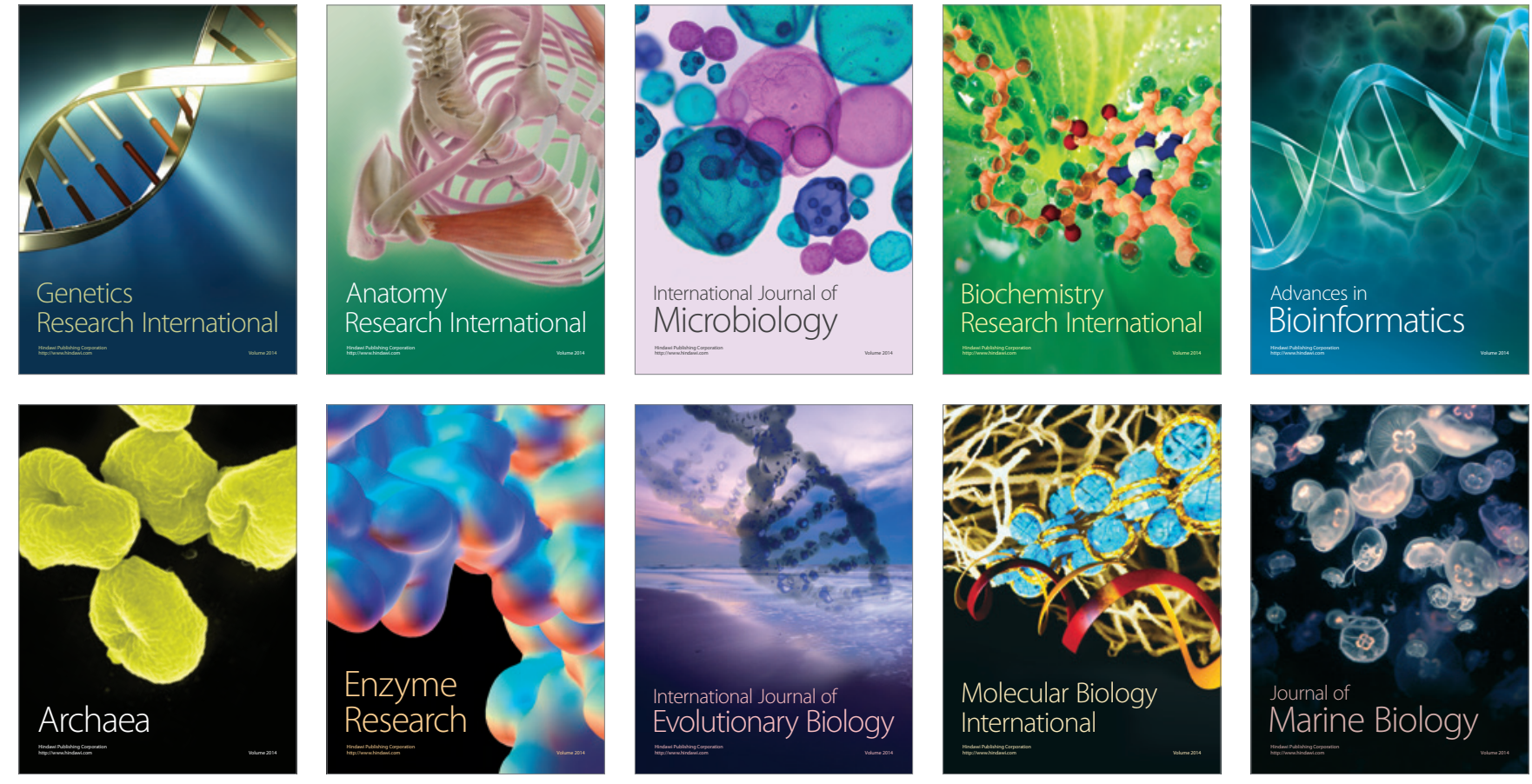